\title{
A study on the association of serum fibroblast growth factor-23 with various indices of chronic kidney disease patients not yet on dialysis
}

\author{
Fatemeh Yaghoubi $^{{ }^{*}}$, Farrokhleghah Ahmadi ${ }^{1}$, Mahboub Lesanpezeshki ${ }^{1}$, Mitra Mahdavi Mazde ${ }^{1}$ \\ ${ }^{1}$ Department of Nephrology, Tehran University Medical Sciences, Tehran, Iran
}

\section{AR T I C L E I N F O}

Article Type:

Original

\section{Article History:}

Received: 28 March 2016

Accepted: 7 April 2016

Published online: 22 April 2016

Keywords:

FGF23

Chronic Kidney Disease

Dyslipidemia

\begin{abstract}
A B S T R A C T
Introduction: Fibroblast growth factor-23 (FGF23) is found as a bone derived hormone which can influence the serum levels of parathormone, phosphorous and 1,25-dihydroxyvitamin D. FGF23 may be a cardiovascular biomarker in patients with chronic kidney disease (CKD). While, FGF23 is inversely correlated with serum adiponectin level, thus it is possible that FGF23 is correlated to fat mass and related to dyslipidemia in CKD.

Objectives: In this investigation, we studied the association of serum levels of FGF23 with serum lipid profile, body mass index (BMI), and various cardiovascular risk factors in patients with CKD.

Patients and Methods: This is a cross-sectional study, which was conducted on 80 nondialysis patients (glomerular filtration rate $[\mathrm{GFR}]<60 \mathrm{cc} / \mathrm{min}$ ) with $\mathrm{CKD}$. Laboratory results and demographic information recorded in pre-defined data sheets and their relationship with FGF23 were analyzed.

Results: Eighty patients were studied. Mean \pm standard deviation (SD) of serum creatinine and FGF23 were $2.18 \pm 0.73 \mathrm{mg} / \mathrm{dl}$ and $15.028 \pm 20.20 \mathrm{RU} / \mathrm{ml}$ respectively. The correlation between FGF23, low-density lipoprotein cholesterol (LDL-C), high-density lipoprotein cholesterol (HDL-C), ApoA, ApoB, intact parathyroid hormone (iPTH), calcium, phosphorous, alkaline phosphatase, 25-hydroxyvitamin D, BMI, waist girth, serum triglyceride and cholesterol was not significant $(P>0.05)$. In this study, the correlations of FGF23, with serum albumin $(P=0.036)$ and systolic blood pressure $(P=0.001)$ were significant.

Conclusion: Our study showed that there are not any significant correlations among FGF23, BMI and lipid profile. There are not any significant correlations between FGF23 and age, sex, and level of calcium, phosphorous, vitamin D. However, the relationships between FGF23 and serum albumin was negatively significant. Additionally we detected a positive significant correlation of FGF23 with level of systolic blood presure.
\end{abstract}

Implication for health policy/practice/research/medical education:

In a study on 80 chronic renal failure patients not yet on dialysis with mean age of $59.82 \pm 14.49$ years, and serum level of fibroblast growth factor-23 (FGF23) of $15.028 \pm 20.20 \mathrm{RU} / \mathrm{ml}$, we found relationships of FGF23 with serum albumin (negative significant correlation) and systolic blood pressure (positive and significant).

Please cite this paper as: Yaghoubi F, Ahmadi F, Lesanpezeshki M, Mahdavi Mazde M. A study on the association of serum fibroblast growth factor-23 with various indices of chronic kidney disease patients not yet on dialysis. J Renal Inj Prev. 2016;5(2):104-107. DOI: 10.15171/jrip.2016.22

\section{Introduction}

The mortality rates in patients with chronic renal failure are higher than the others. Around 50\% of these patients die as a result of cardiovascular disease conditions such as pulmonary edema, chronic heart failure, fatal arrhythmias, pericarditis, valvular disease and cardiogenic shock are some of cardiovascular disorders (CVD), as the leading cause of death in these patients. This can be probably due to a higher prevalence of important risk factors such as hypertension, anemia, uremia, disturbances in calcium, phosphorous and sodium, balance, dyslipidemia, atherosclerosis, diabetes mellitus and smoking. Chronic kidney disease (CKD) has been known as a serious condition which has significant complication. CKD is known as a risk factor of disorders 
such as cardiovascular diseases, electrolyte disturbances, bone disease, cognitive and psychological disorders and many other disease. The nature of this disease is progressive which causes new complications too. On the other hand, there are various parameters which can significantly affect the progress and complications of CKD (1-4).

Terminal stage of chronic renal failure named end-stage renal disease (ESRD). At this stage, patients will need renal replacement therapy. In ESRD patients have lower life expectancy and life quality than general population. Overall mortality rates in these patients are significantly higher than general population. Dialysis and kidney transplantation are two common methods of renal replacement therapy. Availability of the two methods, dialysis quality and support quality for kidney transplantation patients are factors which are strongly related to socio-economic conditions, health care facilities and health care constitutions of the society (5).

\section{Objectives}

In this investigation, we studied the correlation of serum levels of FGF23 with serum lipid profile, BMI and some of the other cardiovascular risk factors in a group of patients with CKD not yet on dialysis.

\section{Patients and Methods}

\section{Study population}

This is a cross-sectional study which was conducted on 80 non-dialysis patients (glomerular filtration rate [GFR] $<60$ cc/min) with CKD.

\section{Data collection}

Patients' demographic information and medical history of the study population including age, sex, height, weight, history of smoking, heart disease, hypertension, diabetes, hyperlipidemia and drug history were obtained and recorded in pre-defined data sheets. Blood pressure of the patients were recorded. Laboratory measurements including serum levels of creatinine, triglyceride, total cholesterol, low-density lipoprotein cholesterol (LDL-C), high-density lipoprotein cholesterol (HDL-C), Apo A, Apo B, fasting blood sugar (FBS), serum levels of calcium, phosphorous, magnesium, alkaline phosphatase, intact parathyroid hormone (iPTH), 25-hydroxyvitamin D level, uric acid and albumin were measured after 12 hours fasting before obtaining their blood specimens, using standard kits. Serum FGF23 was assessed by ELISA method (Demeditec, Germany). Waist girth measured and body mass index (BMI) was also calculated by standard method (NIH).

\section{Exclusion criteria}

The exclusion criteria were using steroids, nonsteroidal anti-inflammatory drugs (NSAIDs) or estrogens. Also presence of active or chronic infection and malignancies were the other exclusion criteria.

\section{Ethical issues}

The research followed the tenets of the Declaration of Helsinki. Informed consent was obtained and the research was approved by the Ethics Committee of Tehran University of
Medical Science (Shariati hospital).

\section{Statistical analysis}

Quantitative variables were calculated as mean \pm standard deviation (SD). $T$ test and Mann-Whitney $U$ test was used for analyzing the serum levels of qualitative variables and chi-square and Fisher's exact test for quantitative variables. $P$ value lower than 0.05 was considered statistically significant. The distribution of quantitative data were assessed by one sample Kolmogorov-Smirnov test. Since FGF23 had not a normal distribution, Spearman's correlation was used for the relation to quantitative variables. Statistical analysis was conducted by SPSS version 18 .

\section{Results}

This study included 44 men (55\%) and 36 women. The mean age of patients was $59.82 \pm 14.49$ years.

Cause of CKD among the patients were hypertension 34 (41\%), diabetes mellitus 18 (21.7\%), glomerulonephritis 10 (12\%), renal stones $4(4.8 \%)$, cysts $2(2.4 \%)$ and unknown 15 (18\%) (Table 1). The mean, SD, minimum and maximum of all variables and correlation of FGF23's variables are shown in Tables 2 and 3. Mean \pm SD of serum creatinine and FGF23 were $2.18 \pm 0.73 \mathrm{mg} / \mathrm{dl}$ and $15.028 \pm 20.20 \mathrm{RU} /$ $\mathrm{ml}$ respectively. Tables 2, 3, and 4 also show the association between FGF23 and other demographic or laboratory parameters. Briefly, correlation of FGF23 with age $(P=0.55)$, serum creatinine $(P=0.19)$, LDL-C $(P=0.063)$, HDL-C $(P=0.168)$ and serum triglyceride levels $(P=0.99)$ was not significant. Likewise, the association of FGF23 serum with serum cholesterol $(P=0.63)$, Apo A $(P=0.21)$, and Apo B $(P=0.74)$ was not significant. Accordingly, the correlation of FGF23 with intact PTH was not significant $(P=0.28)$ (Table 3). The correlation between GFR of all patients $(37.59 \pm 11.21 \mathrm{cc} / \mathrm{min})$ and serum FGF23 was not significant $(P=0.11)$. We categorized GFR into three groups in patients with following prevalence. Group 1 with GFR of 45-59=24 $\mathrm{cc} / \mathrm{min}(29.6 \%)$, group two with GFR of $30-44=34(42 \%)$ and group 3 with GFR of $15-29 \mathrm{cc} / \mathrm{min}=22(27.2 \%)$. None of the groups had correlation with FGF $(P>0.05$; Table 4$)$. In this study, the association of FGF23 with systolic blood pressure was positively significant $(\mathrm{r}=0.41, P=0.001$, Table $4)$ and also with serum albumin $(r=-0.23, P=0.036)$ was negatively significant.

\section{Discussion}

Vitamin D and PTH have traditionally believed as the main factors in bone and mineral hemostasis, however, recent findings regarding FGF23 and bone-kidney have produced a new point of view in CKD and mineral bone disease. In

Table 1. Causes of chronic kidney disease

\begin{tabular}{lc}
\hline Causes & $\mathbf{n}(\%)$ \\
\hline Hypertension & $34(41)$ \\
Diabetes mellitus & $18(21.7)$ \\
Glomerulonephritis & $10(12)$ \\
Renal stones & $4(4.8)$ \\
Cysts & $2(2.4)$ \\
Unknown & $15(18)$ \\
\hline
\end{tabular}


Table 2. Data of the patients

\begin{tabular}{lccc}
\hline Variable & Mean \pm SD & Min. & Max. \\
\hline FGF23 (Ru/ml) & $15.028 \pm 20.20$ & 0.20 & 103.40 \\
Age (y) & $59.82 \pm 14.49$ & 24 & 83 \\
BMI, kg/m² & $27.58 \pm 5.84$ & 17.6 & 40.5 \\
Waist girth (cm) & $96.84 \pm 13.48$ & 62 & 130 \\
FBS (mg/dl) & $105.73 \pm 32.68$ & 50 & 212 \\
GFR (cc/min) & $37.59 \pm 11.21$ & 6.2 & 59 \\
Creatinine (mg/dl) & $2.18 \pm 0.73$ & 1.1 & 5 \\
LDL (mg/dl) & $107.40 \pm 37.58$ & 40 & 230 \\
HDL (mg/dl) & $42.56 \pm 20.20$ & 18 & 167 \\
TG (mg/dl) & $180.66 \pm 100.24$ & 51 & 751 \\
Cholesterol (mg/dl) & $181.16 \pm 47.46$ & 71 & 331 \\
Apo A (g/l) & $1.43 \pm 0.30$ & 0.6 & 2.4 \\
Apo B (g/l) & $0.90 \pm 0.24$ & 0.4 & 1.7 \\
iPTH (pg/ml) & $123.70 \pm 145.58$ & 11.50 & 835 \\
Albumin (g/dl) & $4.20 \pm 44.00$ & 2.8 & 5 \\
Calcium (mg/dl) & $9.01 \pm 0.84$ & 5.4 & 12 \\
Magnesium (mg/dl) & $2.27 \pm 0.33$ & 1.1 & 3 \\
Phosphorous (mg/dl) & $3.96 \pm 0.93$ & 2.10 & 7.20 \\
Alkaline phosphatase (IU/l) & $246.92 \pm 156.66$ & 64 & 972 \\
Uric acid (mg/dl) & $7.46 \pm 1.66$ & 4 & 12.40 \\
BUN (mg/dl) & $81.82 \pm 39.74$ & 27 & 281 \\
\hline 25-hydroxyvitamin D (ng/ml) & $29.95 \pm 18.85$ & 3.7 & 70 \\
\hline SBP (mm Hg) & $132.00 \pm 15.23$ & 110 & 190 \\
DBP (mm Hg) & $79.94 \pm 5.98$ & 65 & 100 \\
\hline Abbreviations: FGF23, & & \\
\hline
\end{tabular}

Abbreviations: FGF23, Fibroblast growth factor 23; iPTH, Intact parathyroid hormone; TG, Triglyceride; HDL, high-density lipoprotein; LDL, low-density lipoprotein; FBS, fasting blood sugar; BMI, body mass index; BUN, blood urea nitrogen; SBP, diastolic blood pressure; DBP, diastolic blood pressure.

$P<0.05$ is considered significant.

summary, the most important function of vitamin D and PTH is stabilization of serum calcium levels in a suitable range through induction of 1,25 -dihydroxy vitamin $\mathrm{D}[1,25$ $(\mathrm{OH}) 2 \mathrm{D} 3$ ] synthesis, leading to reabsorption of calcium and reducing urinary excretion of calcium by kidneys. On the other hand, PTH also increase calcium uptake from bones.

PTH induces production of $1,25(\mathrm{OH})_{2} \mathrm{D}_{3}$ through increasing CYP27b1, increasing reuptake of calcium in proximal tubules and in distal tubules by regulation of TRPV5. Furthermore, PTH increases the uptake of calcium and phosphate in bones through inducing RANKL by osteoblasts (which in turn stimulate osteoclasts that increase uptake from bones). Additionally, increase of 1,25 (OH $)_{2} \mathrm{D}_{3}$ production by kidneys, causes an increase in phosphate and calcium reabsorption by small intestine. Overall, uptake of calcium from bones, decreases in urinary excretion of calcium and increases in its absorption by intestine results to normalizing serum calcium levels. Increasing the uptake of phosphate from bones and by intestines is regulated by PTH and reduces the reuptake of phosphate in renal tubules to balance the levels of phosphate. In fact, FGF23 and bonekidney axis act as a biological route which have recently been discovered as the main players in CKD-mineral and bone disorder (CKD-MBD). Recent findings showed that osteoblasts and osteocytes are important sites for FGF23 production. FGF23 is excreted by bone, targets kidneys,
Table 3. Correlation and significant levels between FGF23 and variables

\begin{tabular}{lll}
\hline Variables & Correlation rate & P \\
\hline FGF23 (Ru/ml) & & \\
Age (y) & 0.067 & 0.55 \\
BMI (Kg/m ${ }^{2}$ ) & -0.036 & 0.75 \\
Waist girth (cm) & 0.15 & 0.18 \\
FBS (mg/dl) & -0.019 & 0.87 \\
GFR (cc/min) & 0.18 & 0.11 \\
Creatinine (mg/dl) & 0.14 & 0.19 \\
LDL (mg/dl) & -0.054 & 0.63 \\
HDL (mg/dl) & -0.156 & 0.163 \\
Triglyceride (mg/dl) & -0.001 & 0.99 \\
Cholesterol (mg/dl) & -0.53 & 0.63 \\
Apo A (g/l) & -0.14 & 0.21 \\
Apo B (g/l) & -0.038 & 0.74 \\
IPTH (pg/ml) & -0.12 & 0.28 \\
Albumin (g/dl) & -0.23 & 0.036 \\
Calcium (mg/dl) & 0.006 & 0.96 \\
Magnesium (mg/dl) & -0.1 & 0.37 \\
Phosphorous (mg/dl) & -0.03 & 0.78 \\
Alkaline phosphatase (IU/L) & 0.038 & 0.74 \\
Uric acid (mg/dl) & -0.064 & 0.57 \\
BUN (mg/dl) & -0.073 & 0.51 \\
\hline 55-hydroxyvitamin D (ng/ml) & -0.19 & 0.33 \\
SBP (mm Hg) & 0.41 & 0.11 \\
\hline DBP (mm Hg) & 0.18 & \\
\hline & & \\
\hline
\end{tabular}

Abbreviations: FGF23, Fibroblast growth factor 23; iPTH, Intact parathyroid hormone; TG, Triglyceride; HDL, high-density lipoprotein; LDL, low-density lipoprotein; FBS, fasting blood sugar; BMI, body mass index; BUN, blood urea nitrogen; SBP, diastolic blood pressure; DBP, diastolic blood pressure.

Table 4. Mean, SD and correlation of GFR to FGF23

\begin{tabular}{lccccc}
\hline GFR & Min. & Max. & Mean \pm SD & Pearson's correlation & $\boldsymbol{P}$ \\
\hline $45-59$ & 0.70 & 91.90 & $12.62 \pm 17.87$ & 0.11 & 0.65 \\
$30-44$ & 0.20 & 40.70 & $11.44 \pm 9.90$ & 0.02 & 0.43 \\
$15-29$ & 0.20 & 103.40 & $21.72 \pm 28.03$ & 0.12 & 0.21 \\
\hline
\end{tabular}

and regulates phosphate and also vitamin D metabolism. It may also have other functions in regulating parathyroid and phosphate which needs to be studied further (6-8). In this study, we assessed the correlation of serum levels of FGF23 and BMI and lipid profile in patients with CKD. We studied 44 men (55\%) and 36 women (45\%). The mean age of patients was $59.82 \pm 14.49$ years and the mean of FGF23 levels was $15.028 \pm 20.20 \mathrm{RU} / \mathrm{ml}$. The correlations of FGF23 with BMI, LDL-C, HDL-C, Apo A, Apo B, triglyceride and cholesterol were not significant. Our study also showed that correlations between FGF23 and other factors such as age, sex, calcium, phosphorous and vitamin D were not significant. Our findings are opposed to the study of Mirza et al, which serum levels of triglyceride and total cholesterol positively correlated with serum FGF23. They also found an inverse relationship between Apo A1 and FGF23 (9). The study of Mirza et al showed that the correlation among FGF23 serum levels and BMI and waist girth is stronger than elevation of triglyceride and reduction of HDL-C, Apo A1. They also found that, serum FGF23 were correlated to 
trunk obesity and total body fat which may reveal a relation between cardiovascular disease and chronic renal failure to the level of FGF23 (9).

Holeck et al showed a correlation between FGF23 and metabolic syndrome in another study. They detected serum value of FGF23 was high in obese and premenopausal women. They concluded that, FGF23 is probably related to the reduction of serum phosphorous in obese patients (10). In animal studies, FGF23 has caused both vascular calcification and glucose and lipid homeostasis disturbances (11). It was also shown that phosphorus reducing therapy with sevelamer in chronic renal failure resulted in lipid improvement including elevation in HDL-C and reduction in LDL$\mathrm{C}$ which can show a relation between bone metabolism and dyslipidemia in CKD. Other families of FGF such as FGF21 have higher levels in obesity, type 2 diabetes and metabolic syndrome (11). In the end-stages of CKD, FGF23 could not reduce serum phosphate and it seems that abnormally high plasma levels of FGF23 cause adverse effects such as LVH, rapid progression of CKD and early mortality (12).

\section{Conclusion}

In our study, only level of blood pressure and serum albumin levels were significantly correlated with FGF23. These require further investigation to better understand of this aspect of CKD patients.

\section{Limitations of the study}

Small proportion of the patients was a limitation of our investigation. Our study was cross-sectional and we could not compare our findings with a control group. Hence, it is suggested to conduct case-control studies with larger sample sizes to better assess the role of FGF23 in renal disease more precisely.

\section{Acknowledgements}

Authors are grateful to research Deputy of Department of Nephrology, Tehran University Medical Sciences, for their financial support of this project.

\section{Conflicts of interest}

The authors report no conflicts of interest. The authors alone are responsible for the content and writing of the article.

\section{Authors' contribution}

All authors contributed to design of the research. PY, FD and SS conducted the research. SS and AP analyzed the data. PS and AP prepared the manuscript. All authors read, revised, and approved the final manuscript.

\section{Ethical considerations}

Ethical issues (including plagiarism, data fabrication, double publication) have been completely observed by the authors.

\section{Funding/Support}

This paper is extracted from thesis of Dr. Fatemeh Yaghoobi and financial support was provided by the Chronic Renal Failure Research Center of Tehran University of Medical Sciences.

\section{References}

1. Shlipak MG, Katz R, Kestenbaum B, Siscovick D, Fried L, Newman A, et al. Rapid decline of kidney function increases cardiovascular risk in the elderly. J Am Soc Nephrol. 2009;20:2625-30. doi: 10.1681/ASN.2009050546.

2. Deo R, Fyr CL, Fried LF, Newman AB, Harris TB, Angleman S, et al. Kidney dysfunction and fatal cardiovascular disease-an association independent of atherosclerotic events: results from the Health, Aging, and Body Composition (Health ABC) study. Am Heart J. 2008;155:62-8. doi: 10.1016/j.ahj.2007.08.012.

3. Bash LD, Erlinger TP, Coresh J, Marsh-Manzi J, Folsom $\mathrm{AR}$, Astor BC. Inflammation, hemostasis, and the risk of kidney function decline in the Atherosclerosis Risk in Communities (ARIC) study. Am J Kidney Dis. 2009;53:596605. doi: 10.1053/j.ajkd.2008.10.044.

4. Campbell RC, Sui X, Filippatos G, Love TE, Wahle C, Sanders PW, et al. Association of chronic kidney disease with outcomes in chronic heart failure: a propensitymatched study. Nephrol Dial Transplant. 2009;24:186-93. doi: $10.1093 / \mathrm{ndt} / \mathrm{gfn} 445$.

5. Mailloux LU, Napolitano B, Bellucci AG, Mossey RT, Vernace MA, Wilkes BM. The impact of co-morbid risk factors at the start of dialysis upon the survival of ESRD patients. ASAIO J. 1996;42:164-9.

6. Jones G, Strugnell SA, DeLuca HF. Current understanding of the molecular actions of vitamin D. Physiol Rev. 1998;78:1193-231.

7. Yamashita T, Yoshioka M, Itoh N. Identification of a novel fibroblast growth factor, FGF-23, preferentially expressed in the ventrolateral thalamic nucleus of the brain. Biochem Biophys Res Commun. 2000;277:494-8. doi: 10.1006/ bbrc.2000.3696.

8. de Groot T, Lee K, Langeslag M, Xi Q, Jalink K, Bindels RJ, et al. Parathyroid hormone activates TRPV5 via PKA-dependent phosphorylation. J Am Soc Nephrol. 2009;20:1693-704. doi: 10.1681/ASN.2008080873.

9. Mirza MA, Larsson A, Lind L, Larsson TE. Circulating fibroblast growth factor- 23 is associated with vascular dysfunction in the community. Atherosclerosis. 2009;205:38590. doi: 10.1016/j.atherosclerosis.2009.01.001.

10. Holecki M, Chudek J, Więcek A, Titz-Bober M, Duława J. The serum level of fibroblast growth factor-23 and calcium-phosphate homeostasis in obese perimenopausal women. Int J Endocrinol. 2011;2011: 707126. doi. org/10.1155/2011/707126.

11. Li H, Bao Y, Xu A, Pan X, Lu J, Wu H, et al. Serum fibroblast growth factor 21 is associated with adverse lipid profiles and gamma-glutamyltransferase but not insulin sensitivity in Chinese subjects. J Clin Endocrinol Metab. 2009;94:2151-6. doi: 10.1210/jc.2008-2331.

12. Jüppner H. Phosphate and FGF23. Kidney Int Suppl. 2011;:S24-7. doi: 10.1038/ki.2011.27.

Copyright ( $\odot 2016$ The Author(s); Published by Nickan Research Institute. This is an open-access article distributed under the terms of the Creative Commons Attribution License (http://creativecommons.org/licenses/by/4.0), which permits unrestricted use, distribution, and reproduction in any medium, provided the original work is properly cited. 